\title{
RADIOTHERAPY IN OSTEOSARCOMAS
}

doi: 10.2478/rojost-2018-0042

\section{R. Mitrică, L. Şerbănescu, O. Trifănescu}

"Carol Davila" University of Medicine and Pharmacy, Bucharest, Romania

Osteosarcoma represents a rare primary tumor of the bone, but it ranks $5^{\text {th }}$ among children and adolescents. When diagnosed, many patients already have a disseminated disease at subclinical level. Current treatment includes surgery and chemotherapy. Radiotherapy is not commonly indicated, especially given the radioresistant characteristic of this type of cancer and it is usually administered for residual disease after surgery, inoperable tumors or in palliation, such as whole lung irradiation.

The purpose of this paper was to identify the real contribution of radiotherapy in osteosarcomas, with solutions to improve local control and survival.

We reviewed clinical studies, starting with the period before chemotherapy, in order to identify treatment techniques related criteria that may influence local control, survival, and secondary toxicity. Modern radiotherapy techniques are outlined with their dosimetric advantages. Following the 1990s, the combination between proper surgery and chemotherapy has eliminated the need for pre-operative radiotherapy. Most clinical studies have used adjuvant radiotherapy for patients with positive resection margins, with good results but hard to dissociate from those of the concurrently administered chemotherapy. Proton therapy is frequently indicated for these patients, due to the healthy tissue sparing characteristics. Studies have pointed out the correlation between radiotherapy response and tumor size, total administered dose, chemo sensitivity. Whole lung irradiation for patients with lung metastases is inferior to chemotherapy. Radiotherapy associated complications include joint fibrosis with reduced functional capabilities, bone fracture, allograft loss and carcinogenic risk.

Currently, radiotherapy is not the first choice when treating osteosarcomas, but new evidence is emerging for combining modern techniques, such as proton therapy with surgery and chemotherapy for patients with incomplete resections or inoperable tumors. Keywords: osteosarcoma, radiotherapy, radioresistant, proton therapy 